University of Wollongong

Research Online

Faculty of Engineering - Papers (Archive)

Faculty of Engineering and Information

Sciences

$1-1-2007$

\title{
Distributed MIMO systems using Asterism decoding
}

Phillip Conder

Victoria University

Jason Gao

Victoria University

Mehran Abolhasan

University of Wollongong, mehran.abolhasan@uts.edu.au

Follow this and additional works at: https://ro.uow.edu.au/engpapers

Part of the Engineering Commons

https://ro.uow.edu.au/engpapers/3517

\section{Recommended Citation}

Conder, Phillip; Gao, Jason; and Abolhasan, Mehran: Distributed MIMO systems using Asterism decoding 2007, 269-272.

https://ro.uow.edu.au/engpapers/3517

Research Online is the open access institutional repository for the University of Wollongong. For further information contact the UOW Library: research-pubs@uow.edu.au 


\section{Distributed MIMO systems using Asterism decoding}

\author{
Phillip Conder, Jason Gao \\ Centre for Telecommunications and Microelectronics \\ Victoria University, \\ Melbourne, Australia \\ Email: \{Phillip.Conder, Jason.Gao\}@vu.edu.au
}

\author{
Mehran Abolhasan \\ Telecommunications and IT Research Institute \\ University of Wollongong, \\ Wollongong, Australia \\ Email: mehrana@uow.edu.au
}

\begin{abstract}
Multiple Input Multiple Output (MIMO) systems have received enormous attention by researchers as they can provide a roughly linear increase in capacity of wireless communications systems by using multiple transmit and receive antennas. Even with the larger raw data rates provided by MIMO, the throughput of these systems can be limited by the number of users (or networks) sharing the medium.

This paper shows how multiple users (or networks) transmitting simultaneously can be represented as a MIMO system with a lager number of transmit than receive antennas. After reviewing existing decoders for such a MIMO system, this paper then proposes applying an Asterism based decoder to distinguish between multiple users transmitting simultaneously. This produces a new multi-user access scheme that can be applied in addition to a primary access method, such as CSMA-CA.
\end{abstract}

\section{INTRODUCTION}

The area of Multiple Input Multiple Output (MIMO) communications systems has received enormous attention as they can provide a roughly linear increase [1] in data rate by using multiple transmit and receive antennas. Incorporating a multiple antenna system with error correcting code, such as Turbo coding, is a popular approach to achieving increased data rates [2].

At the same time as increasing data rates, future mobile communications are promised to be small, lightweight mobile devices which present problems to the implementation of multiple antenna systems. Thus reducing the size and cost of mobile terminals by reducing the number receive antennas will be factor in the implementation of MIMO especially for larger number of transmit antennas.

Of the existing MIMO decoding schemes for MIMO systems, such as Maximum Likelihood (ML), Zero Forcing (ZF), Bell Labs Layered Space-Time (BLAST) [3] and Sphere decoding [2], only Maximum Likelihood and Sphere decoding can successfully decode systems with a larger number of transmit than receive antennas. This is at the detriment of computational complexity. Asterism decoding [4] is a scheme that achieved Maximum Likelihood performance for MIMO systems by considering the larger complex constellation created by a multiple transmit antennas and a single receive antenna. It was then extended to operate in combination with Sphere decoding to reduce the computational complexity of MIMO decoding for any number of transmit and receive antennas.
This paper proposes applying an Asterism based decoder to effectively produce an additional multi-user access scheme that could be applied in addition to an existing access scheme. The paper is ordered as follows: Section 2 describes Multiple Input Multiple Output systems and previous decoders such as Maximum Likelihood and Sphere decoder for systems with a larger number of transmit than receive antennas $\left(n_{t}>n_{r}\right)$. Section 3 reviews Asterism based decoders for such MIMO systems with $n_{t}>n_{r}$, while Section 4 gives a brief system description of a Multiple User Access scheme that uses multi path fading to distinguish between users and can be represented as a MIMO system with $n_{t}>n_{r}$. Finally Section 5 shows the performance of uncoded and Turbo coded Multi-User MIMO systems decoding using an Asterism based decoder.

\section{Multiple InPut Multiple Output Systems}

The Multiple Input Multiple Output approach was first introduced by Lucent's Bell Labs, with their BLAST family of Space Time Code structures [3]. A Turbo encoded Vertical Bell Laboratories Layered Space-Time (VBLAST) scheme is considered in this paper. An incoming bitstream is combined with forward error correction information by a Turbo encoder, mapped onto symbols from a complex constellation before demultiplexed into substreams. Let $n_{t}$ be the number of transmit antennas, $n_{r}$ be the number of receive antennas, and $s=$ $\left(s_{1}, s_{2}, \ldots s_{n_{t}}\right)^{T}$ denote the vector of symbols transmitted in one symbol period. The received vector $R=\left(R_{1}, R_{2} \ldots R_{n_{r}}\right)^{T}$ becomes:

$$
R=H s+n
$$

where $n=\left(n_{1}, n_{2}, \ldots n_{n_{r}}\right)^{T}$ is the noise vector of additive white Gaussian noise (AWGN) of variance $\sigma^{2}$ equal to $\frac{1}{2}$ per dimension.

The $n_{r} \times n_{t}$ channel matrix produced between each transmit and receive antenna:

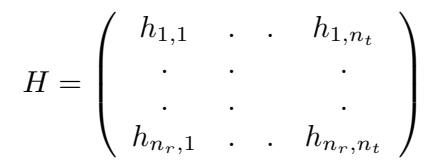

contains independent identical distribution (i.i.d.) complex fading gains $h_{i, j}$ from the $j^{\text {th }}$ transmit antenna to the $i^{\text {th }}$ receive antenna. We assume flat fading where the magnitude of the elements of $H$ have a Rayleigh distribution. 


\section{A. Existing MIMO decoders for $n_{t}>n_{r}$}

There has been considerable research for decoding MIMO systems, almost all scheme concentrate on systems with the same or more receive antennas than transmit antennas.

Optimal Maximum Likelihood decoding is achieved by minimising

$$
\|H s-R\|^{2}
$$

for all elements of $s$, which are symbols of constellation of size $C$. This would produce a search of length $C^{n_{t}}$. A system using $n_{t}=4$ and $16 Q A M$ would have to test each of the 65536 possibilities, far beyond being easily implemented on low cost and low power hardware [5].

The principle of Sphere decoding is to search the closest lattice point to the received vector within a sphere centered at zero forcing estimate of the symbols $\widetilde{s}$. The Sphere decoder described in [2] decomposes the channel matrix and received vector from complex numbers into its real and imaginary parts to produce a channel matrix of twice the size of the original. The multi-dimensional Sphere decoder now becomes an interval centered around $\widetilde{s}$ and a Cholesky factorisation of $H$ is also used to determine the interval size based on the starting radius and any previously decoded symbols.

The Sphere decoder is essentially the same for $n_{t}>n_{r}$ but uses the Padded channel matrix $H_{\text {pad }}$ to not only determine $\widetilde{s}$ but also the Cholesky factorisation. While the padding of $H$ is satisfactory for the calculation of $\widetilde{s}$, when used for the Cholesky factorisation, the padded $H$ produces very small numbers for the lowest $n_{t}-n_{r}$ levels of the triangular matrix. This means that the Sphere decoder has a complexity of the order of $C^{n_{t}-n_{r}}$ i.e. Sphere decoder decomposes to the complexity of the Maximum Likelihood detection scheme when $n_{r}=1$.

\section{Asterism DeCODING}

Asterism decoding, proposed in [4], was created to reduce the computational complexity of Maximum Likelihood decoding and retain the performance and flexibility of reducing the number of receive antennas. This is achieved by considering all possible transmitted symbol combinations created by a multiple transmit antennas and a single receive antenna. When all the possible combinations are plotted onto a complex plane a larger complex constellation is formed which can be divided or grouped into Asterisms. Asterism decoding uses these grouping to determine the Maximum Likelihood solution without having to test every point of the complex constellation.

The large complex constellation generated for a systems with $n(t)=4$, QPSK and random channel coefficientsts is shown in Figure 1. It can be seen that all 256 points of the large complex constellation are contained within the four largest Asterisms. Each of the largest outer Asterisms can be divided into four smaller Asterisms which in turn can be grouping into four more Asterisms.

For ease of explanation, we make the assumption that the magnitude of $H$ in (2) is decreasing i.e. $\left|h_{1}\right|$ is the largest and $\left|h_{4}\right|$ is the smallest. In implementing an Asterism decoding,

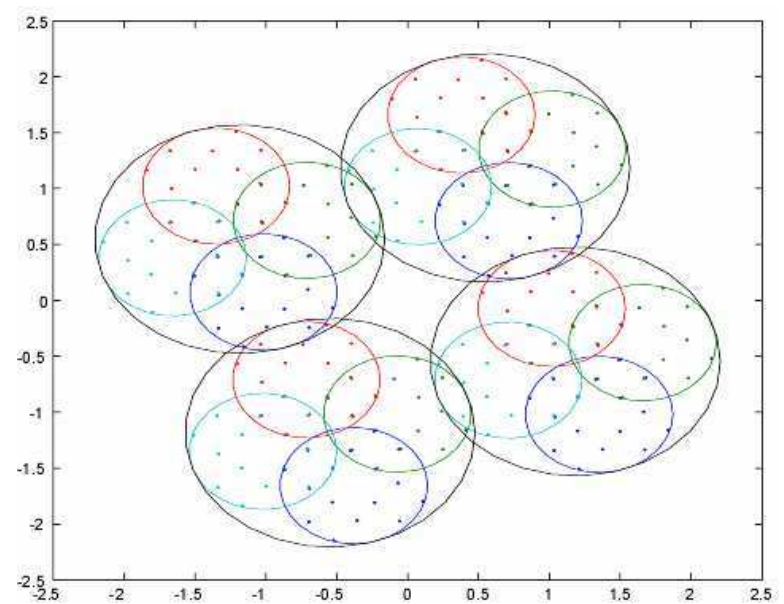

Fig. 1. Large constellation generated by grouped into 16 Asterisms

this simlpy requires the algorithm to reorder the detection order so the symbol with the largest channel magnitude is detected first and the symbol with smallest channel magnitude is detected last. The radius of the Asterism radius at detection stage $k$ is:

$$
\operatorname{Radius}_{(k)}=\beta \sum_{j=k+1}^{n_{t}}|h(j)|
$$

where $\beta=$ largest symbol magnitude, which for 16QAM is $\sqrt{18}$ the magnitude for the symbols $[3+3 i,-3+3 i,-3-3 i, 3-$ $3 i]$. These Asterisms at the first detection stage are centered at $h_{1} \times s_{i}$. Every possible combination is covered by these Asterism circles. The size and the amount of overlap of these circles is determined by the number of transmit antennas, the magnitude of the elements of $H$ and the Hamming distance of the constellation.

If the received vector $R$ is inside the one or more circles it is possibly the Maximum Likelihood solution. The algorithm then subtracts this possible solution from $R$ and determines whether modified $R$ is in one of the new Asterism circles centered at $h_{2} \times s_{i}$ and of radius $\left|h_{3}\right|$. This recursive process continues until all $n_{t}$ symbols are found. If there is more than one combination found, the combination with the lowest complex distance measurement is chosen to be the ML solution.

When noise places the received vector outside any of the Asterisms the algorithm will find no symbol combination and fail. This can happen not only at the first stage of decoding, where noise puts $R$ outside the area covered by the largest Asterisms, but also at any later stage of decoding where $R$ may be part of a larger Asterism but not part of a small Asterism of later stages of decoding.

To overcome this the decoding the algorithm must not only find which Asterism the received vector is inside, but also allow for the case where it is inside none of the Asterisms. In this case the decoding chooses the Asterism to which it is 

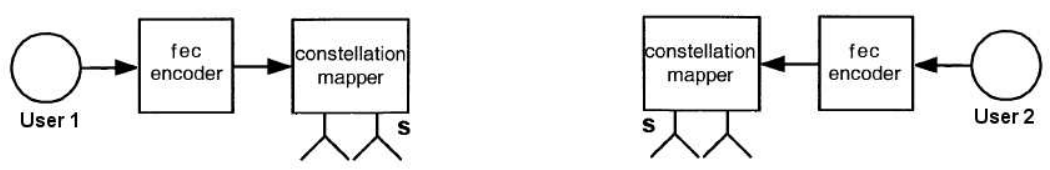

H
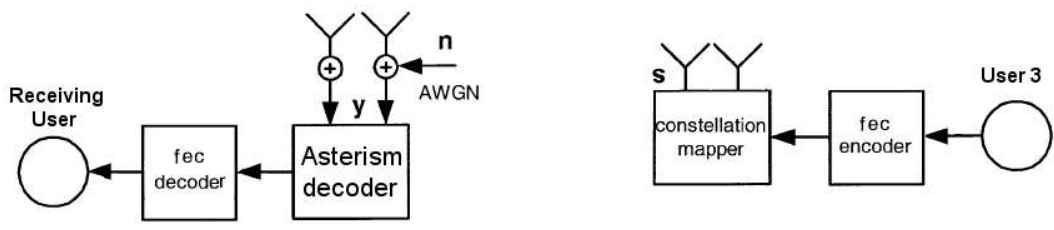

Fig. 2. Simplified block diagram of the Multiuser Access scheme, where each user has Multiple Transmit and Receive antennas.

closest to and continues the process to find the ML solution.

While using Asterism decoding to multiple transmit and a single receiver antenna system produces the ML performance, the performance of this type of system is relatively poor. To overcome this the use of multiple antennas at the receive was considered. The received vector where $n_{t}=4$ and $n_{r}=2$ becomes:

$$
\left[\begin{array}{l}
R_{1} \\
R_{2}
\end{array}\right]=\left[\begin{array}{llll}
h_{1,1} & h_{1,2} & h_{1,3} & h_{1,4} \\
h_{2,1} & h_{2,2} & h_{2,3} & h_{2,4}
\end{array}\right]\left[\begin{array}{l}
s_{1} \\
s_{2} \\
s_{3} \\
s_{4}
\end{array}\right]+\left[\begin{array}{l}
n_{1} \\
n_{2}
\end{array}\right]
$$

To take advantage of the information provided by additional antennas Maximum Ratio combining is used at each stage of decoding and was found to have near Maximum Likelihood performance.

The complexity of Asterism decoding with multiple receive antennas was further reduced by combining the Sphere and Asterism decoders [6]. The proposal implemented an Asterism decoder to detect the first $n_{t}-n_{r}$ symbols which are then passed to a Sphere decoder to find the remaining symbols. The Sphere decoder algorithm described in Section 2 applied to a real system where symbols from a real lattice are found, this proposal utilised a variation of the complex Sphere decoder [2] to allow it be easily integrated with the Asterism decoder

\section{Distributed Mimo as a Multi-User Access SCHEME}

A simplified block diagram of a simple multi-user system using MIMO described in this paper is shown in Figure 2. Each of the users has $n_{t}=n_{r}=2$ antennas and including Forward Error Correction encoding/decoding. In a traditional time sharing multiple access system, when a user (say User 1) transmits its message to the receiver, the remaining users (Users 2 and 3) not only cannot transmit to the receiver, but also cannot transmit a message to any other user within range of the receiving user. Normally if more than one user transmits simultaneously the the signals will interfere with each other causing an error in transmission. If we assume that the users are separated enough so that their fading coefficients can be considered uncorrelated (similar to the main assumption of MIMO systems), then the channel matrix of $n$ users transmitting simultaneously becomes:

$$
H=\left[\begin{array}{llll}
H_{u 1} & H_{u 2} & \cdot H_{u n}
\end{array}\right]
$$

where $H_{u 1}$ is a $n_{t} \times n_{r}$ channel matrix (in this example a $2 \times 2$ matrix) between each of the users' transmit antennas to the receiver's antennas.

A multiuser MIMO system with 2 users transmitting simultaneously will be equivalent to the MIMO system of $n_{t}=4$ and $n_{r}=2$ describe in Section 2. Equation (5) now becomes:

$\left[\begin{array}{l}R_{1} \\ R_{2}\end{array}\right]=\left[\begin{array}{llll}h_{u 1_{1,1}} & h_{u 1_{2,1}} & h_{u 2_{1,1}} & h_{u 2_{2,1}} \\ h_{u 1_{1,2}} & h_{u 1_{2,2}} & h_{u 2_{1,2}} & h_{u 2_{2,2}}\end{array}\right]\left[\begin{array}{l}s_{u 1_{1}} \\ s_{u 1_{2}} \\ s_{u 2_{1}} \\ s_{u 2_{2}}\end{array}\right]+\left[\begin{array}{c}n_{1} \\ n_{2}\end{array}\right]$

Similarly, a system with 3 users transmitting simultaneously would be equal to a MIMO system with 6 transmit and 2 receive antennas and with 4 users would be equivalent to a MIMO with 8 transmit and 2 receive.

As described in Section III, an Asterism based decoder can successfully decode such a MIMO system with more transmit than receive antennas, hence applying such a decoder to such a system with multiple transmitting users, creates a Multi-User access scheme in addition to existing time sharing scheme often used in Mobile Ad-Hoc networks.

\section{Simulation Results}

In this section we provide simulation results, by Monte Carlo methods, to illustrate the performance of an Asterism decoded Multi-User Access scheme describe in Section IV. The channels are assumed fast flat-fading, i.e. they are the constant over a single data burst and change from burst to 


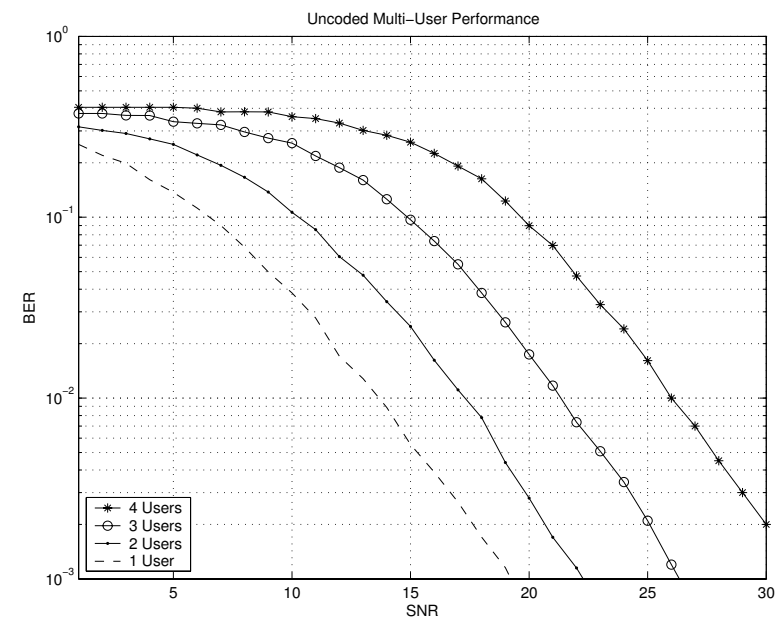

Fig. 3. Performance comparison of Multiuser Asterism decoding for $n_{t}=2$, $n_{r}=2$ for each user using QPSK without error coding.

burst, this could be due to the movement of nodes, and the fading coefficients are generated according to a Rayleigh distribution. The assumption is made that the users' transmissions are synchronized but no other implementation of high layers is made at this stage.

Figure 3 shows the performance of an Asterism decoded Multi-User MIMO system with one, two, three and four simultaneous transmitting users and that the performance of the system reduce by approximately $4 d B$ for each additional user transmitting simultaneously. The loss between four transmitting users and a single user is approximately $12 d B$. This may seem quite poor at first, but considering that without an Asterism based decoder at the receiver, only one user could transmit at a time producing congestion in the network and producing a higher BER when multiple users transmit simultaneously.

Figure 4 shows the performance of Turbo coded Multi-User MIMO system with one, two, three and four simultaneous transmitting users. All the simulations used a frame of length 9216 bits, two $(7,5)$ convolution coders with puncturing giving a total rate of $1 / 2$ and all results are for 5 iterations of the Turbo decoder. The Turbo decoder used in our simulations consisted of two Log-MAP decoders [7] permuted by a deinterleaver. The Turbo coded MIMO system produces a BER of $10^{-5}$ at a SNR of $10 d B$ and again each additional of a user requires an increases in $S N R$ of $4 d B$ to produce the same BER. For example two users require $14 d B$, three users requires $18 d B$ and four users requires approximately $22 d B$ to achieve a BER of $10^{-5}$.

\section{CONCLUSION}

This paper proposed applying an Asterism based decoder to produce an additional multi user access scheme on top of the primary access method. By applying an Asterism based decoder and hence the possibility of an additional multi user

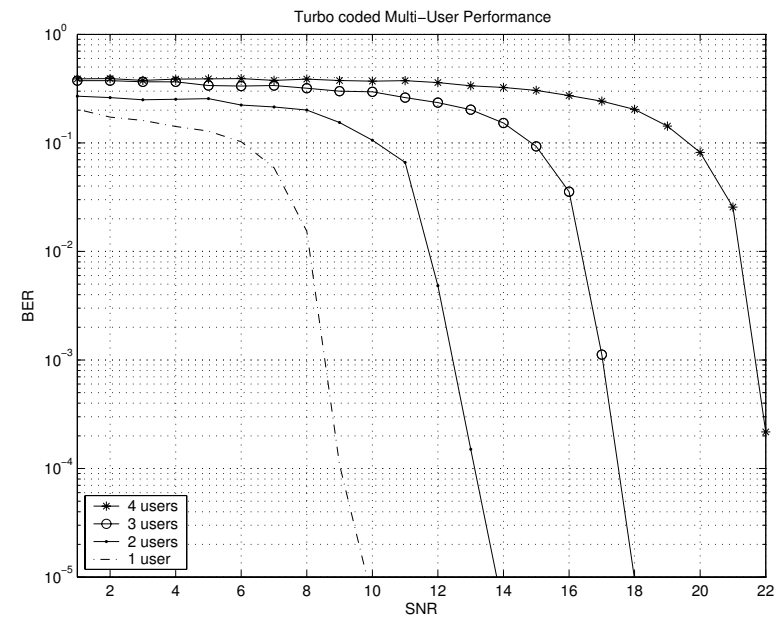

Fig. 4. Performance comparison of Multiuser Asterism decoding for $n_{t}=2$, $n_{r}=2$ for each user using QPSK with Turbo error coding.

access scheme, multiple transmissions from different users can be decoded successfully, potentially increasing the efficiency and throughput systems such as Mobile Ad Hoc Networks [8].

Further avenues of research include investigating methods that take advantage of the Multi-User access scheme, synchronisation methods and increasing the performance beyond what has been presented in this paper. If increasing the computational complexity was considered, further research could include reducing the $4 d B$ loss produced by each additional user. This could be achieved by a combination of more powerful error correction codes, interleaver design and joint symbol detection and error detection in an iteractive manner.

\section{REFERENCES}

[1] E. Telatar, "Capacity of Multi-antenna Gaussian Channels", AT \& T Bell Labs, Murray Hill, NJ, Tech. Rep., 1995.

[2] B. Hochwald and S. ten Brink, "Achieving Near-Capacity on a MultipleAntenna Channel", IEEE Transactions on Communications, Vol 51, No. 3, March 2003.

[3] G.J. Foschini, "Layered Space-Time Architecture for Wireless Communications in a Fading Environment when using Multiple Antennas", Bell Labs Technical Journal, Autumn 1996.

[4] P. Conder and T. A. Wysocki, "Asterism decoding for Layered SpaceTime systems using 8PSK", 2004 IEEE Sarnoff Symposium, Princeton, New Jersey, USA, 27-28 April 2004.

[5] D. Garrett, L. Davis, G. Woodward, "19.2 Mbit/s 4 /spl times/ 4 BLAST/MIMO detector with soft ML outputs", Electronics Letters , Volume: 39 Issue: 2 , 23 Jan. 2003.

[6] P. Conder and T. A. Wysocki, "Asterism decoding for Turbo coded MIMO systems", IEEE 61st Vehicular Technology Conference, Stockholm, Sweden, May 30 - June 12005.

[7] L. Hanzo, T. Liew, and B. Yeap, "Turbo Coding, Turbo Equalisation and Space-Time Coding for transmission over fading channels", volume 1 ., 2002, John Wiley and Sons Ltd.

[8] M.Abolhasan, T.A.Wysocki, and E.Dutkiewicz "A Review of Routing Protocols for Mobile Ad hoc Networks", In Elsevier Journal of Ad hoc Networks, 2 (2004), 1-22. 